\title{
Coupled-cluster method: A lattice-path-based subsystem approximation scheme for quantum lattice models
}

\author{
R. F. Bishop and P. H. Y. Li \\ School of Physics and Astronomy, Schuster Building, University of Manchester, Manchester M13 9PL, UK
}

(Received 9 December 2010; published 29 April 2011)

\begin{abstract}
An approximation hierarchy, called the lattice-path-based subsystem (LPSUBm) approximation scheme, is described for the coupled-cluster method (CCM). It is applicable to systems defined on a regular spatial lattice. We then apply it to two well-studied prototypical (spin- $\frac{1}{2}$ Heisenberg antiferromagnetic) spin-lattice models, namely, the $X X Z$ and the $X Y$ models on the square lattice in two dimensions. Results are obtained in each case for the ground-state energy, the ground-state sublattice magnetization, and the quantum critical point. They are all in good agreement with those from such alternative methods as spin-wave theory, series expansions, quantum Monte Carlo methods, and the CCM using the alternative lattice-animal-based subsystem (LSUBm) and the distance-based subsystem (DSUBm) schemes. Each of the three CCM schemes (LSUBm, DSUBm, and LPSUB $m$ ) for use with systems defined on a regular spatial lattice is shown to have its own advantages in particular applications.
\end{abstract}

DOI: 10.1103/PhysRevA.83.042111

PACS number(s): 03.65.Fd, 03.65.Ge, 75.10.Jm, 11.10.Ef

\section{INTRODUCTION}

The coupled-cluster method (CCM) [1-9] is widely recognized nowadays as providing one of the most powerful, most universally applicable, and numerically most accurate, at attainable levels of computational implementation, of all available $a b$ initio methods of microscopic quantum manybody theory. The number of successful applications of the $\mathrm{CCM}$ to a wide range of physical and chemical systems is now impressively large. Some typical examples, from among many others, of systems existing in the spatial continuum, and to which the method has been applied, include the electron gas [10-12], atomic nuclei and nuclear matter [13,14], and molecules [15]. In these and many other cases the numerical results obtained with the CCM are either the best or among the best available. For the case of the electron gas, for example, which is still one of the most intensely studied of all quantum many-body systems, the CCM results [12] for the correlation energy agree over the entire metallic density range to within less than one millihartree per electron (i.e., better than 1\%) with the essentially exact Green's function Monte Carlo results available for this system [16]. More recently and more relevantly for the present discussion, the CCM has also been very successfully applied to systems on a discrete spatial lattice, such as spin-lattice models of quantum magnetism [17-29].

One of the features of the CCM, in which it differs from many other techniques for dealing with quantum many-body systems, is that, if required, it deals from the outset with infinite systems. Thus, one never needs to take explicitly the limit $N \rightarrow \infty$, where $N$ is the number of interacting particles or the number of lattice sites. On the other hand, of course, the method does require us to make approximations for its implementation. These typically involve making selections for which terms to include in the cluster expansions for the correlation operators that are intrinsic to the way the method parametrizes the many-body wave functions, as we describe more fully in Sec. II below.

We and our collaborators have developed previously several efficient and systematic approximation schemes for the CCM that are specifically geared to use with lattice systems [18,30-33]. The most widely used and the most successful such CCM approximation schemes for spin-lattice systems up to now have been the so-called LSUB $m$ and SUB $n-m$ schemes discussed in detail below in Sec. IV. The LSUBm scheme in particular has been demonstrated on many occasions to be highly accurate in practice for a wide variety of strongly correlated spin systems. Of special importance is the fact that the scheme seems to be equally applicable to both frustrated and unfrustrated systems, with comparable levels of accuracy attained in both cases. Nevertheless, a disadvantage of the LSUB $m$ scheme is that the number of spin configurations retained at a given level in describing the many-body correlations present in the wave functions rises very rapidly (and typically superexponentially) with the truncation index $m$. Since we typically then have to take the limit $m \rightarrow \infty$ numerically to obtain estimates for exact physical properties of the system, it is desirable to have calculations at as many values of the truncation index $m$ as possible.

This one drawback of the prevailing LSUB $m$ scheme has led us recently to develop an alternative scheme, the so-called DSUB $m$ scheme [33]. A primary aim of any such scheme should be that in practical applications of it one is able to implement more levels of approximation (i.e., to use more values of the index $m$ ) than in the corresponding $\operatorname{LSUB} m$ scheme for the same problem. In this way one thus has more data points available for the necessary $m \rightarrow \infty$ extrapolations, for calculated physical quantities, to the exact limit where all spin configurations are retained in the many-body wave functions. A second very desirable feature of any such scheme is that it also captures the physically most important multispin configurations in the system wave functions at relatively low orders in the index $m$, so that physical properties converge more rapidly as $m$ is increased.

Although the recently developed DSUB $m$ scheme [33] partially met the above criteria, there is no doubt that users of the CCM would still welcome more choices of approximation schemes. In that context the principal aim of the present work is to outline a further such scheme that we now denote as the 
LPSUB $m$ scheme, and which is also specifically designed for use with lattice systems. The scheme is motivated on physical grounds, and its merits are illustrated by applications to some stereotypical models that have been well studied previously by other techniques, including the CCM itself but with other approximation schemes.

The general formalism of the CCM is first briefly outlined in Sec. II, after which we discuss its specific applications to systems confined to the sites of a regular spatial lattice in Sec. III. In Sec. IV we first describe the existing CCM truncation schemes for spin-lattice systems, and then motivate and describe the LPSUB $m$ scheme. The accuracy of this scheme in practice is then illustrated by applying it to two wellstudied antiferromagnetic spin-lattice models [20,22], namely, the spin-half $X X Z$ and $X Y$ models on the two-dimensional (2D) square lattice. Both models contain a free parameter in the Hamiltonian which, as it is varied, carries the zero-temperature models through a quantum phase transition at some critical value of this parameter. Both models have previously been the subject of CCM studies, using the LSUBm and DSUBm truncation schemes, to calculate the ground-state (gs) energy and gs order parameter (which, in the present cases, is the sublattice magnetization).

We note that all microscopic techniques applied to infinite spin-lattice problems need to be extrapolated in terms of some appropriate parameter. For example, for such main alternative methods to the CCM as the exact diagonalization of small clusters and quantum Monte Carlo simulations of larger clusters, the extrapolation parameter is the number of lattice sites $N$. As previously noted, one huge advantage of the CCM is that it exactly preserves the Goldstone linkedcluster theorem, and hence size extensivity, at all levels of approximation. Hence we may (and do) work in the limit of infinite lattice size $(N \rightarrow \infty)$ from the very beginning. By contrast, the extrapolations for the CCM are done in terms of some truncation index $m$, where in the limit $m \rightarrow \infty$ we retain all possible spin configurations in the wave functions of the system, and the calculations become formally exact. The extrapolation schemes used in practice [22,31,34-36] are themselves also first described in Sec. V. The LPSUBm scheme is then applied to the spin-half $X X Z$ model and the spin-half $X Y$ model, both on the 2D square lattice, in Secs. VI and VII, respectively. Results are compared critically with those from corresponding CCM studies using the alternative LSUB $m$ and DSUB $m$ schemes, as well as with the best results from other methods. We conclude in Sec. VIII with a summary and discussion of our main findings.

\section{REVIEW OF THE CCM FORMALISM}

We first briefly describe the CCM formalism. The interested reader is referred, for example, to Refs. [8,9] for further details. In any application of the CCM a first step is to choose a normalized model (or reference) state $|\Phi\rangle$ that can act as a cyclic vector with respect to a complete set of mutually commuting multi-configurational creation operators $C_{I}^{+} \equiv\left(C_{I}^{-}\right)^{\dagger}$. The index $I$ here is a set index that labels and uniquely identifies the many-particle configuration created in the state $C_{I}^{+}|\Phi\rangle$. The exact ket and bra gs energy eigenstates
$|\Psi\rangle$ and $\langle\tilde{\Psi}|$ of the many-body system are then parametrized in the CCM form as

$$
\begin{aligned}
& |\Psi\rangle=e^{S}|\Phi\rangle, \quad S=\sum_{I \neq 0} \mathcal{S}_{I} C_{I}^{+}, \\
& \langle\tilde{\Psi}|=\langle\Phi| \tilde{S} e^{-S}, \quad \tilde{S}=1+\sum_{I \neq 0} \tilde{\mathcal{S}}_{I} C_{I}^{-},
\end{aligned}
$$

where

$$
H|\Psi\rangle=E|\Psi\rangle, \quad\langle\tilde{\Psi}| H=E\langle\tilde{\Psi}|,
$$

where we have defined $C_{0}^{+} \equiv 1 \equiv C_{0}^{-}$. The requirements on the multi-configurational creation operators are that any manyparticle state can be written exactly and uniquely as a linear combination of the states $\left\{C_{I}^{+}|\Phi\rangle\right\}$, which hence fulfill the completeness relation

$$
\sum_{I} C_{I}^{+}|\Phi\rangle\left\langle\Phi\left|C_{I}^{-}=1=\right| \Phi\right\rangle\left\langle\Phi\left|+\sum_{I \neq 0} C_{I}^{+}\right| \Phi\right\rangle\langle\Phi| C_{I}^{-},
$$

together with the conditions

$$
\begin{aligned}
C_{I}^{-}|\Phi\rangle & =0=\langle\Phi| C_{I}^{+}, \quad \forall I \neq 0, \\
{\left[C_{I}^{+}, C_{J}^{+}\right] } & =0=\left[C_{I}^{-}, C_{J}^{-}\right] .
\end{aligned}
$$

In practice approximations are necessary to restrict the label set $I$ to some finite (e.g., LSUBm) or infinite (e.g., $\mathrm{SUB} n$ ) subset, as described more fully below. The correlation operator $S$ is a linked-cluster operator and is decomposed in terms of a complete set of creation operators $C_{I}^{+}$. When acting on the model state it creates excitations that are correlated cluster states. Although the manifest Hermiticity, $(\langle\tilde{\Psi}|)^{\dagger} \equiv$ $|\Psi\rangle /\langle\Psi \mid \Psi\rangle$, is lost, the normalization conditions $\langle\tilde{\Psi} \mid \Psi\rangle=$ $\langle\Phi \mid \Psi\rangle=\langle\Phi \mid \Phi\rangle \equiv 1$ are preserved. The CCM Schrödinger Eqs. (3) are thus written as

$$
H e^{S}|\Phi\rangle=E e^{S}|\Phi\rangle, \quad\langle\Phi| \tilde{S} e^{-S} H=E\langle\Phi| \tilde{S} e^{-S},
$$

and their equivalent similarity-transformed forms become

$$
e^{-S} H e^{S}|\Phi\rangle=E|\Phi\rangle, \quad\langle\Phi| \tilde{S} e^{-S} H e^{S}=E\langle\Phi| \tilde{S} .
$$

The static gs CCM correlation operators $S$ and $\tilde{S}$ contain the real $c$-number correlation coefficients $\mathcal{S}_{I}$ and $\tilde{\mathcal{S}}_{I}$ that need to be calculated. Clearly, once the coefficients $\left\{\mathcal{S}_{I}, \tilde{\mathcal{S}}_{I}\right\}$ are known, all other gs properties of the many-body system can be derived from them. Thus, the gs expectation value of an arbitrary operator $A$, for example, can be expressed as

$$
\bar{A} \equiv\langle A\rangle \equiv\langle\tilde{\Psi}|A| \Psi\rangle=\left\langle\Phi\left|\tilde{S} e^{-S} A e^{S}\right| \Phi\right\rangle \equiv A\left(\mathcal{S}_{I}, \tilde{\mathcal{S}}_{I}\right)
$$

The gs correlation coefficients $\left\{\mathcal{S}_{I}, \tilde{\mathcal{S}}_{I}\right\}$ are now found by simply inserting the parametrizations of Eqs. (1) and (2) into the similarity-transformed Schrödinger Eqs. (8) and projecting onto the complete sets of states $\left\{\langle\Phi| C_{I}^{-}\right\}$and $\left\{C_{I}^{+}|\Phi\rangle\right\}$, respectively,

$$
\begin{aligned}
\left\langle\Phi\left|C_{I}^{-} e^{-S} H e^{S}\right| \Phi\right\rangle & =0, \quad \forall I \neq 0, \\
\left\langle\Phi\left|\tilde{S}\left(e^{-S} H e^{S}-E\right) C_{I}^{+}\right| \Phi\right\rangle & =0, \quad \forall I \neq 0 .
\end{aligned}
$$

By pre-multiplying the ket-state Eqs. (8) with the state $\langle\Phi| \tilde{S} C_{I}^{+}$ and using the commutation relation (6) it is easy to show that Eq. (11) may be rewritten in the form

$$
\left\langle\Phi\left|\tilde{S} e^{-S}\left[H, C_{I}^{+}\right] e^{S}\right| \Phi\right\rangle=0, \quad \forall I \neq 0 .
$$


Equations (10)-(12) may be equivalently derived by requiring that the gs energy expectation value, $\bar{H} \equiv\langle\tilde{\Psi}|H| \Psi\rangle=$ $\left\langle\Phi\left|\tilde{S} e^{-S} H e^{S}\right| \Phi\right\rangle$, is minimized with respect to the entire set $\left\{\mathcal{S}_{I}, \tilde{\mathcal{S}}_{I}\right\}$. In practice we thus need to solve Eqs. (10) and (12) for the set $\left\{\mathcal{S}_{I}, \tilde{\mathcal{S}}_{I}\right\}$. We note that Eqs. (9) and (10) show that the gs energy at the stationary point has the simple form

$$
E \equiv E\left(\mathcal{S}_{I}\right)=\left\langle\Phi\left|e^{-S} H e^{S}\right| \Phi\right\rangle,
$$

which also follows immediately from the ket-state Eq. (8) by projecting it onto the state $\langle\Phi|$. It is important to note, however, that this (bi-)variational formulation does not necessarily lead to an upper bound for $E$ when the summations over the index set $\{I\}$ for $S$ and $\tilde{S}$ in Eqs. (1) and (2) are truncated, due to the lack of manifest Hermiticity when such approximations are made. Nevertheless, as we have pointed out above, one can prove [9] that the important Hellmann-Feynman theorem is preserved in all such approximations.

Equations (10) now represents a coupled set of multinomial equations for the $c$-number correlation coefficients $\left\{\mathcal{S}_{I}\right\}$. The well-known nested commutator expansion of the similaritytransformed Hamiltonian,

$$
e^{-S} H e^{S}=H+[H, S]+\frac{1}{2 !}[[H, S], S]+\cdots,
$$

and the fact that all of the individual components of $S$ in the decomposition of Eq. (1) commute with one another by construction [and see Eq. (6)] together imply that each element of $S$ in Eq. (1) is linked directly to the Hamiltonian in each of the terms in Eq. (14). Thus, each of the coupled Eqs. (10) is of Goldstone linked-cluster type, thereby also guaranteeing that all extensive variables, such as the energy, scale linearly with particle number $N$. Thus, at any level of approximation obtained by truncation in the summations on the index $I$ in the parametrizations of Eqs. (1) and (2), we may (and, in practice, do) work from the outset in the limit $N \rightarrow \infty$ of an infinite system.

It is now also important for practical applications to note that each of the seemingly infinite-order (in $S$ ) linked-cluster Eqs. (10) will actually be of finite length when expanded using Eq. (14). The reason for this is that the otherwise infinite series in Eq. (14) will actually terminate at a finite order, provided only (as is usually the case, including those for the Hamiltonians considered in this paper) that each term in the Hamiltonian $H$ contains a finite number of singleparticle destruction operators defined with respect to the reference (or generalized vacuum) state $|\Phi\rangle$. In this way the CCM parametrization naturally leads to a workable scheme, that can be implemented computationally in an efficient manner, to evaluate the set of configuration coefficients $\left\{\mathcal{S}_{I}, \tilde{\mathcal{S}}_{I}\right\}$ by solving the coupled sets of Eqs. (10) and (12), once we have devised practical and systematic truncation hierarchies for limiting the set of multi-configurational set indices $\{I\}$ to some suitable finite or infinite subset. We turn our attention to such truncation schemes in Sec. IV after first reviewing the application of the method (described in general terms above) to the specific case of spin-lattice systems.

\section{REVIEW OF THE CCM FOR SPIN-LATTICE SYSTEMS}

We now briefly describe how the general CCM formalism outlined in Sec. II is implemented for spin-lattice problems in practice. As we have already asserted is the case for any application of the CCM to a general quantum many-body system, a first step is to choose a suitable reference state $|\Phi\rangle$ in which the state of the spin (viz., in practice, its projection onto a specific quantization axis in spin space) on every lattice site $k$ is characterized. The choice of $|\Phi\rangle$ will clearly depend on both the system being studied and, more importantly, which of its possible phases is being considered. We describe examples of such choices later for the particular models that we utilize here as test cases for our truncation scheme.

We note first that, whatever choice for $|\Phi\rangle$ is made, it is very convenient to treat the spins on every lattice site in an arbitrarily given model state $|\Phi\rangle$ as being equivalent, in order to create as universal a methodology as possible. A suitably simple way of doing so is to introduce a different local quantization axis and a correspondingly different set of spin coordinates on each lattice site $k$, so that all spins, whatever their original orientation in $|\Phi\rangle$ in the original global spin-coordinate system, align along the same direction (which, in order to be definite, we henceforth choose as the negative $z$ direction) in these local spin-coordinate frames. In practice this can always be done by defining a suitable rotation in spin space of the global spin coordinates at each lattice site $k$. Such rotations are canonical transformations that leave unchanged the fundamental spin commutation relations,

$$
\begin{gathered}
{\left[s_{k}^{+}, s_{k^{\prime}}^{-}\right]=2 s_{k}^{z} \delta_{k k^{\prime}}, \quad\left[s_{k}^{z}, s_{k^{\prime}}^{ \pm}\right]= \pm s_{k}^{ \pm} \delta_{k k^{\prime}},} \\
s_{k}^{ \pm} \equiv s_{k}^{x} \pm i s_{k}^{y},
\end{gathered}
$$

among the usual SU(2) spin operators $\mathbf{s}_{k} \equiv\left(s_{k}^{x}, s_{k}^{y}, s_{k}^{z}\right)$ on lattice site $k$. Each spin has a total spin quantum number $s_{k}$, where $\mathbf{s}_{k}^{2}=s_{k}\left(s_{k}+1\right)$ is the $\mathrm{SU}(2)$ Casimir operator. For the models considered here, $s_{k}=s=\frac{1}{2}$, at every lattice site $k$.

It is clear that after the local spin axes have been chosen as described above, the model state thus has all spins pointing downward (i.e., in the negative $z$ direction, where $z$ is the quantization axis),

$$
|\Phi\rangle=\bigotimes_{k=1}^{N}|\downarrow\rangle_{k}, \quad \text { in the local spin axes, }
$$

here $|\downarrow\rangle \equiv|s,-s\rangle$ in the usual $\left|s, m_{s}\right\rangle$ notation for single spin states.

The configuration indices $I$ now simply become a set of lattice site indices, $I \rightarrow\left(k_{1}, k_{2}, \ldots, k_{m}\right)$, and in the local spin frames defined above the corresponding generalized multiconfigurational creation operators $C_{I}^{+}$thus become simple products of single spin-raising operators, $C_{I}^{+} \rightarrow s_{k_{1}}^{+} s_{k_{2}}^{+} \cdots s_{k_{m}}^{+}$. Thus, for example, the ket-state CCM correlation operator is expressed as

$$
S=\sum_{m=1}^{N} \sum_{k_{1} k_{2} \cdots k_{m}} \mathcal{S}_{k_{1} k_{2} \cdots k_{m}} s_{k_{1}}^{+} s_{k_{2}}^{+} \cdots s_{k_{m}}^{+},
$$

and $\tilde{S}$ is similarly defined in terms of the spin-lowering operators $s_{k}^{-}$. Since the operator $S$ acts on the state $|\Phi\rangle$, in which all spins point along the negative $z$-axis in the local 
spin-coordinate frames, every lattice site $k_{i}$ in Eq. (18) can be repeated up to no more than $2 s$ times in each term where it is allowed, since a spin $s$ has only $(2 s+1)$ possible projections along the quantization axis.

The allowed configurations are often further constrained in practical applications by symmetries in the problem and by conservation laws. An example of the latter is provided by the $X X Z$ model considered below in Sec. VI, for which we can easily show that the total $z$-component of spin, $s_{z}^{T}=\sum_{k=1}^{N} s_{k}^{z}$, in the original global spin coordinates, is a good quantum number since $\left[s_{T}^{z}, H\right]=0$ in this case. Finally, for the quasiclassical magnetically ordered states that we calculate here for the models in both Secs. VI and VII, the order parameter is the sublattice magnetization $M$, which is given within the local spin coordinates defined above as

$$
M \equiv-\frac{1}{N}\left\langle\tilde{\Psi}\left|\sum_{k=1}^{N} s_{k}^{z}\right| \Psi\right\rangle=-\frac{1}{N} \sum_{k=1}^{N}\left\langle\Phi\left|\tilde{S} e^{-S} s_{k}^{z} e^{S}\right| \Phi\right\rangle .
$$

The similarity-transformed Hamiltonian $\bar{H} \equiv e^{-S} H e^{-S}$, and all of the corresponding matrix elements in Eqs. (9)-(13) and (19), for example, may then be evaluated in the local spin coordinate frames by using the nested commutator expansion of Eq. (14), the commutator relations of Eq. (15), and the simple universal relations

$$
\begin{array}{r}
s_{k}^{-}|\Phi\rangle=0, \quad \forall k, \\
s_{k}^{z}|\Phi\rangle=-\frac{1}{2}|\Phi\rangle, \quad \forall k,
\end{array}
$$

that hold at all lattice sites in the local spin frames.

\section{CCM APPROXIMATION SCHEMES}

When all many-body configurations $I$ are included in the $S$ and $\tilde{S}$ operators in Eqs. (1) and (2) the CCM formalism is exact. In practice, however, it is necessary to use approximation schemes to truncate the correlation operators. The main approximation scheme used to date for continuous systems is the so-called SUBn scheme described below. For systems defined on a regular periodic spatial lattice, we have a further set of approximation schemes which are based on the discrete nature of the lattice, such as the SUB $n-m, \operatorname{LSUB} m$, and DSUB $m$ schemes described below. The various schemes and their definitions for spin-lattice systems are as follows:
(1) the $\mathrm{SUB} n$ scheme, in which only the correlations involving $n$ or fewer spin-raising operators for $S$ are retained, but with no further restrictions on the spatial separations of the spins involved in the configurations;

(2) the SUB $n-m$ scheme which includes only the subset of all $n$-spin-flip configurations in the $\mathrm{SUB} n$ scheme that are defined over all lattice animals of size $\leqslant m$, where a lattice animal is defined as a set of contiguous lattice sites, each of which is nearest neighbor to at least one other in the set; and

(3) the LSUBm scheme, which includes all possible multispin-flip configurations defined over all lattice animals of size $\leqslant m$. The LSUB $m$ scheme is thus equivalent to the SUB $n-m$ scheme with $n=2 \mathrm{sm}$, for particles of spin quantum number $s$. For example, for spin- $\frac{1}{2}$ systems, for which no more than one spin-raising operator, $s_{k}^{+}$, can be applied at each site $k$, $\mathrm{LSUB} m \equiv \mathrm{SUB} m-m$.

(4) the DSUBm scheme, which is defined to include in the correlation operator $S$ all possible configurations of spins involving spin-raising operators where the maximum length or distance of any two spins apart is defined by $L_{m}$, where $L_{m}$ is a vector joining sites on the lattice and the index $m$ labels lattice vectors in order of size. Hence DSUB1 includes only nearest-neighbor pairs, etc.

We now turn our attention to the LPSUB $m$ scheme that uses real paths on the lattice to determine the fundamental spin configurations. For the LPSUB $m$ scheme, we measure distances $P_{m}$ along the sides of the lattice, rather than the distance $L_{m}$ used in the DSUB $m$ scheme. For example, for a square lattice, we restrict the size of the square-lattice plaquette (i.e., the size of the array) by the longest path $\left(P_{m}\right)$ between particles in the array,

$$
P_{m}=k+l, \quad m \equiv k+l,
$$

where $k$ and $l$ are the sides of the lattice plaquette in the $x$ and $y$ directions. Table I illustrates the formulation of the spin-array configurations retained in the LPSUB $m$ scheme at the $m$ th level of approximation for a 2D square lattice. Similar tables can be constructed for an arbitrary regular lattice in any number of dimensions. It shows, for example, that the LPSUB5 approximation on a 2D square lattice involves all clusters of spins (and their associated spin-raising operators) for which the real path distance between any two spins is less than or equal to 5 (lattice spacings). Clearly the LPSUB $m$ and the DSUB $m$ schemes both order the multispin configurations

TABLE I. Illustration of the formulation of the spin-array configurations retained in the LPSUB $m$ scheme on a square lattice at the $m$ th level of approximation, in terms of lattice increments $k$ and $l$ along the two sides of the square lattice [note that the number of spins on plaquette $k \times l$ is $(k+1) \times(l+1)$ ].

\begin{tabular}{lcc}
\hline \hline LPSUB $m$ & $\begin{array}{c}\text { Size of Square-Lattice Rectangular Plaquette or Size of Array } \\
k \times l\left(\text { with } P_{m}=k+l\right)\end{array}$ & $\begin{array}{c}\text { Maximum No. } \\
\text { of Spins }\end{array}$ \\
\hline LPSUB1 & $1 \times 0$ & 2 \\
LPSUB2 & LPSUB1 $+1 \times 1$ & 4 \\
LPSUB3 & LPSUB2 $+3 \times 0+2 \times 1$ & 6 \\
LPSUB4 & LPSUB3 $+4 \times 0+3 \times 1+2 \times 2$ & 9 \\
LPSUB5 & LPSUB4 $+5 \times 0+4 \times 1+3 \times 2$ & 12 \\
LPSUB6 & LPSUB5 $+6 \times 0+5 \times 1+4 \times 2+3 \times 3$ & 16 \\
LPSUB7 & LPSUB6 $+7 \times 0+6 \times 1+5 \times 2+4 \times 3$ & 20 \\
\hline \hline
\end{tabular}


in terms, roughly, of their compactness, whereas the LSUBm scheme orders them, roughly, according to the overall size of the lattice animals (or polyominoes), defined as the number of contiguous lattice sites involved.

\section{CCM EXTRAPOLATION SCHEMES}

Each of the above truncated approximations clearly becomes exact when all possible multispin cluster configurations are retained, i.e., in the limit as $n \rightarrow \infty$ and/or $m \rightarrow \infty$. We have considerable experience, for example, with the appropriate extrapolations for the LSUBm scheme [22,31,34-36], which shows that the gs energy behaves in the large- $m$ limit as a power series in $1 / \mathrm{m}^{2}$, whereas the order parameter $M$ behaves as a power series in $1 / m$ (at least for relatively unfrustrated systems). Initial experience with the new LPSUB $m$ scheme shows that it behaves similarly with the scaling laws

$$
E / N=a_{0}+a_{1}\left(\frac{1}{m^{2}}\right)+a_{2}\left(\frac{1}{m^{2}}\right)^{2},
$$

for the gs energy $(E / N)$, and

$$
M=b_{0}+b_{1}\left(\frac{1}{m}\right)+b_{2}\left(\frac{1}{m}\right)^{2},
$$

for the staggered magnetization $(M)$, respectively, as we show in more detail below for the two examples of the spin- $\frac{1}{2} X X Z$ and $X Y$ models on the 2D square lattice.

In order to fit well to any fitting formula that contains $n$ unknown parameters, one should always have at least $(n+1)$ data points for a robust and stable fit, and in all our CCM calculations in practice we try our best to obey this primary edict, in so far as it is possible to do so with the available computing power. In so far as is possible we also try to avoid using the least approximate data points (e.g., LSUBm, SUB $m-m$, DSUB $m$ points with $m \leqslant 2$ ) since these low- $m$ data points are rather far from the corresponding large- $m$ limits. In the ensuing discussion we refer to this as our secondary edict. Nevertheless, we do include such points if it is necessary to do so to preserve our above primary edict. In these latter cases, however, we are always careful to do some other careful consistency checks on the robustness and accuracy of our results.

In the next two sections we now illustrate the use and power of the LPSUBm scheme by applying it to two prototypical spin-half models defined on the 2D square lattice, namely, the $X X Z$ model in Sec. VI and the $X Y$ model in Sec. VII.

\section{THE SPIN- $\frac{1}{2}$ ANTIFERROMAGNETIC $X X Z$ MODEL ON THE SQUARE LATTICE}

As an illustration of the use of the LPSUBm scheme we first consider its application to the spin- $\frac{1}{2} X X Z$ model on the infinite square lattice. The Hamiltonian of the $X X Z$ model, in global spin coordinates, is written as

$$
H_{X X Z}=\sum_{\langle i, j\rangle}\left[s_{i}^{x} s_{j}^{x}+s_{i}^{y} s_{j}^{y}+\Delta s_{i}^{z} s_{j}^{z}\right],
$$

where the sum on $\langle i, j\rangle$ runs over all nearest-neighbor pairs of sites on the lattice and counts each pair only once. Since the square lattice is bipartite, we consider $N$ to be even, so that each sublattice contains $\frac{1}{2} N$ spins, and we consider only the case where $N \rightarrow \infty$. The Néel state is the ground state (GS) in the trivial Ising limit $\Delta \rightarrow \infty$, and a phase transition occurs at $\Delta=1$. Indeed, the classical GS demonstrates perfect Néel order in the $z$ direction for $\Delta>1$, and a similar perfectly ordered $x-y$ planar Néel phase for $-1<\Delta<1$. For $\Delta<-1$ the classical GS is a ferromagnet.

The case $\Delta=1$ is equivalent to the isotropic Heisenberg model, whereas $\Delta=0$ is equivalent to the isotropic version of the $X Y$ model considered in Sec. VII below. The $z$ component of total spin, $s_{T}^{z}$, is a good quantum number as it commutes with the Hamiltonian of Eq. (25). Thus one may readily check that $\left[s_{T}^{z}, H_{X X Z}\right]=0$. Our interest here is in those values of $\Delta$ for which the GS is an antiferromagnet.

The CCM treatment of any spin system is initiated by choosing an appropriate model state $|\Phi\rangle$ (for a particular regime), so that a linear combination of products of spin-raising operators can be applied to this state and all possible spin configurations are determined. There is never a unique choice of model state $|\Phi\rangle$. Clearly our choice should be guided by any physical insight that we can bring to bear on the system or, more specifically, to that particular phase of it that is under consideration. In the absence of any other insight into the quantum many-body system it is common to be guided by the behavior of the corresponding classical system (i.e., equivalently, the system when the spin quantum number $s \rightarrow \infty)$. The $X X Z$ model under consideration provides just such an illustrative example. Thus, for $\Delta>1$ the classical Hamiltonian of Eq. (25) on the 2D square lattice (and, indeed, on any bipartite lattice) is minimized by a perfectly antiferromagnetically Néel-ordered state in the spin $z$ direction. However, the classical gs energy is minimized by a Néel-ordered state with spins pointing along any direction in the spin $x-y$ plane (say, along the spin $x$ direction) for $-1<\Delta<1$. Either of these states could be used as a CCM model state $|\Phi\rangle$ and both are likely to be of value in different regimes of $\Delta$ appropriate to the particular quantum phases that mimic the corresponding classical phases. For present illustrative purposes we restrict ourselves to the $z$ aligned Néel state as our choice for $|\Phi\rangle$, written schematically as $|\Phi\rangle=|\cdots \downarrow \uparrow \downarrow \uparrow \cdots\rangle$, in the global spin axes, where $|\uparrow\rangle \equiv$ $\left|\frac{1}{2},+\frac{1}{2}\right\rangle$ and $|\downarrow\rangle \equiv\left|\frac{1}{2},-\frac{1}{2}\right\rangle$ in the usual $\left|s, m_{s}\right\rangle$ notation. Such a state is, clearly, likely to be a good starting point for all $\Delta>1$, down to the expected phase transition at $\Delta=1$ from a $z$-aligned Néel phase to an $x-y$ planar Néel phase.

As indicated in Sec. III it is now convenient to perform a rotation of the axes for the up-pointing spins (i.e., those on the sublattice with spins in the positive $z$ direction) by $180^{\circ}$ about the spin $y$-axis, so that $|\Phi\rangle$ takes the form given by Eq. (17). Under this rotation, the spin operators on the original up sublattice are transformed as

$$
s^{x} \rightarrow-s^{x}, \quad s^{y} \rightarrow s^{y}, \quad s^{z} \rightarrow-s^{z} .
$$




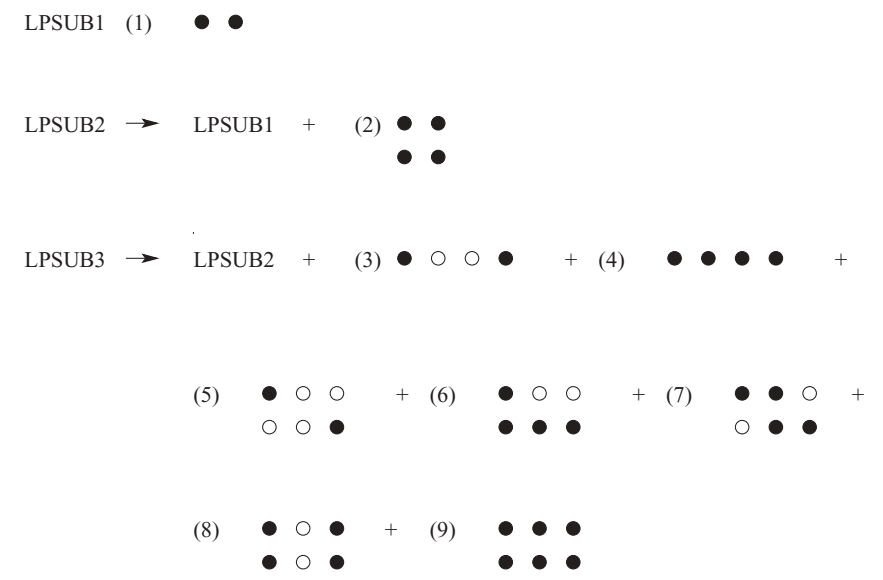

FIG. 1. The fundamental configurations for the LPSUB $m$ scheme with $m=\{1,2,3\}$ for the spin- $\frac{1}{2} X X Z$ model on a square lattice in two dimensions. The filled circles mark the relative positions of the sites of the square lattice on which the spins are flipped with respect to the model state. The unfilled circles represent unflipped sites.

The Hamiltonian of Eq. (25) may thus be rewritten in these local spin coordinate axes as

$$
H_{X X Z}=-\frac{1}{2} \sum_{\langle i, j\rangle}\left[s_{i}^{+} s_{j}^{+}+s_{i}^{-} s_{j}^{-}+2 \Delta s_{i}^{z} s_{j}^{z}\right] .
$$

As in any application of the CCM to spin-lattice systems, we now include in our approximations at any given order only those fundamental configurations that are distinct under the point and space group symmetries of both the lattice and the Hamiltonian. The number $N_{f}$ of such fundamental configurations at any level of approximation may be further restricted whenever additional conservation laws come into play. For example, in our present case, the $X X Z$ Hamiltonian of Eq. (25) commutes with the total uniform magnetization, $s_{T}^{z}=\sum_{k=1}^{N} s_{k}^{z}$, in the global spin coordinates, where $k$ runs over all lattice sites. The GS is known to lie in the $s_{T}^{z}=0$ subspace, and hence we exclude configurations with an odd number of spins or with unequal numbers of spins on the two equivalent sublattices of the bipartite square lattice. We show in Fig. 1 the fundamental configurations that are accordingly allowed for the LPSUB $m$ approximations for this spin- $\frac{1}{2} X X Z$ model on the 2D square lattice, with $1 \leqslant m \leqslant 3$. We see, for example, that $N_{f}=9$ at the LPSUB3 level of approximation.

The LPSUB $m$ approximations can readily be implemented for the present spin- $\frac{1}{2} X X Z$ model on the 2D square lattice for all values $m \leqslant 6$ with reasonably modest computing power. By comparison, the LSUB $m$ and DSUB $m$ schemes can both be implemented with comparable computing resources for all values $m \leqslant 9$. Numerical results for the gs energy per spin and the sublattice magnetization are shown in Table II at the isotropic point $\Delta=1$ at various levels of approximation, and corresponding results for the same quantities are displayed graphically in Figs. 2 and 3 as functions of the anisotropy parameter $\Delta$.

We also show in Table II for the isotropic Heisenberg Hamiltonian $(\Delta=1)$ the results for the gs energy and sublattice magnetization using the (quadratic) extrapolation schemes of Eqs. (23) and (24), respectively, of the LPSUB $m$

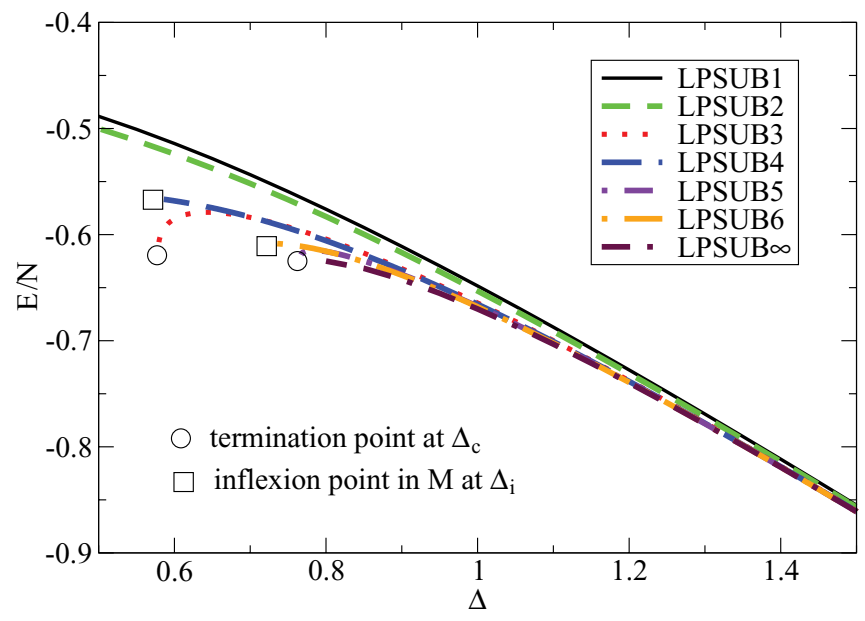

FIG. 2. (Color online) CCM results for the ground-state energy per spin, $E / N$, as a function of the anisotropy parameter $\Delta$, of the spin- $\frac{1}{2} X X Z$ model on the $2 \mathrm{D}$ square lattice, using various LPSUB $m$ approximations based on the $z$-aligned Néel model state. The LPSUB $m$ results with $m=\{2,4,6\}$ are extrapolated using the quadratic fit of Eq. (23) and shown as the curve LPSUB $\infty . \Delta_{i} \equiv$ magnetization point of inflexion, described in the text.

data, employing various subsets of results. Comparison is also made with corresponding LSUB $m[37,38]$ and DSUB $m$ [33] extrapolation schemes for the same model. The results are generally observed to agree very well with each other. Excellent agreement of all the CCM extrapolations is also obtained with the results from the best of the alternative methods for this model, including third-order spin-wave theory (SWT) [39], linked-cluster series expansion (SE) techniques [40], the extrapolations to infinite lattice size $(N \rightarrow \infty)$ from the exact diagonalization (ED) of small lattices [41], and quantum Monte Carlo (QMC) calculations for larger lattices

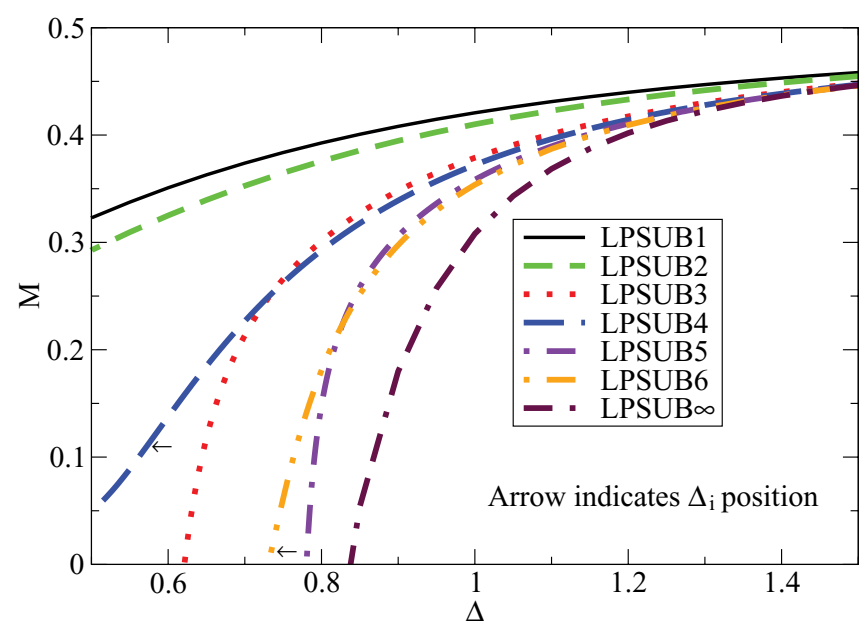

FIG. 3. (Color online) CCM results for the ground-state sublattice magnetization, $M$, as a function of the anisotropy parameter $\Delta$, of the spin- $\frac{1}{2} X X Z$ model on the $2 \mathrm{D}$ square lattice, using various LPSUB $m$ approximations based on the $z$-aligned Néel model state. The LPSUB $m$ results with $m=\{2,4,6\}$ are extrapolated using the quadratic fit of Eq. (24) and shown as the curve LPSUB $\infty . \Delta_{i} \equiv$ point of inflexion in the curve, shown by arrows in the figure. 
TABLE II. The ground-state energy per $\operatorname{spin}(E / N)$ and sublattice magnetization $(M)$ for the spin- $\frac{1}{2} X X Z$ model on the $2 \mathrm{D}$ square lattice, obtained using the CCM LPSUB $m$ approximation scheme with $1 \leqslant m \leqslant 6$ at $\Delta=1 . N_{f}$ is the number of fundamental configurations at a given LPSUB $m, \mathrm{LSUB} m$, or DSUB $m$ level of approximation. $\Delta_{i} \equiv \operatorname{LPSUB} m$ sublattice magnetization point of inflexion. The LPSUB $m$ results for odd values of $m$, even values of $m$, and the whole series of $m$ are extrapolated separately. These results are compared to calculations using third-order spin-wave theory (SWT) [39], series expansions (SE) [40], exact diagonalization (ED) [41], quantum Monte Carlo (QMC) [42], LSUB $\infty$ extrapolations of the CCM LSUB $m$ approximations $[22,37,38]$, and the DSUB $\infty$ extrapolations of the CCM DSUB $m$ approximations [33].

\begin{tabular}{|c|c|c|c|c|c|c|}
\hline Method & $N_{f}$ & $\begin{array}{c}E / N \\
(\Delta=1)\end{array}$ & $\begin{array}{c}M \\
(\Delta=1)\end{array}$ & $\Delta_{i}$ & $\Delta_{c}$ & $\begin{array}{c}\text { Max. No. } \\
\text { of Spins }\end{array}$ \\
\hline LPSUB1 & 1 & -0.64833 & 0.421 & & & 2 \\
\hline LPSUB2 & 2 & -0.65311 & 0.410 & 0.258 & & 4 \\
\hline LPSUB3 & 9 & -0.66442 & 0.379 & & 0.579 & 6 \\
\hline LPSUB4 & 35 & -0.66565 & 0.372 & 0.586 & & 8 \\
\hline LPSUB5 & 265 & -0.66761 & 0.358 & & 0.766 & 12 \\
\hline LPSUB6 & 2852 & -0.66807 & 0.354 & 0.735 & & 16 \\
\hline LSUB8 & 1287 & -0.66817 & 0.352 & & 0.844 & 8 \\
\hline LSUB10 & 29605 & -0.66870 & 0.345 & & & 10 \\
\hline SWT & & -0.66999 & 0.3069 & & & \\
\hline SE & & -0.66930 & 0.307 & & & \\
\hline $\mathrm{ED}$ & & -0.67000 & 0.317 & & & \\
\hline QMC & & $-0.669437(5)$ & $0.3070(3)$ & & & \\
\hline \multicolumn{6}{|c|}{ Extrapolation } & Based on \\
\hline LPSUB $\infty$ & & -0.66953 & 0.320 & & & $m=\{1,3,5\}$ \\
\hline LPSUB $\infty$ & & -0.67004 & 0.308 & 1.093 & & $m=\{2,4,6\}$ \\
\hline LPSUB $\infty$ & & -0.66867 & 0.328 & & & $2 \leqslant m \leqslant 6$ \\
\hline LPSUB $\infty$ & & -0.67107 & 0.288 & & & $3 \leqslant m \leqslant 6$ \\
\hline DSUB $\infty$ & & -0.67082 & 0.308 & 1.009 & & $m=\{6,8,10\}$ \\
\hline $\operatorname{LSUB} \infty$ & & -0.67029 & 0.304 & & & $m=\{3,5,7,9\}$ \\
\hline $\operatorname{LSUB} \infty$ & & -0.66966 & 0.310 & & & $m=\{4,6,8,10\}$ \\
\hline LSUB $\infty$ & & -0.66962 & 0.308 & & & $m=\{6,8,10\}$ \\
\hline
\end{tabular}

[42]. Interestingly, these high-precision QMC data have been used by Löw [43] in connection with exact inequalities of Kennedy, Lieb, and Shastry [44] to prove rigorously the existence of Néel order for this 2D Heisenberg model.

As discussed in Sec. V we always prefer to have at least 4 LPSUB $m$ calculations with different values of the truncation index $m$, to fit to the three unknown parameters of the quadratic fitting expressions for $E / N$ and $M$ in Eqs. (23) and (24). This primary edict is not violated if we extrapolate the LPSUB $m$ data using both odd and even values of $m$ in the range $2 \leqslant m \leqslant 6$ or $3 \leqslant m \leqslant 6$. We note, however, that if we extrapolate using only the three even values $m=\{2,4,6\}$ or using the three odd values $m=\{1,3,5\}$ then we violate both the primary and secondary edicts discussed above. Nevertheless, the extrapolated results using the even set $m=\{2,4,6\}$ are seen to be in good agreement with those from the alternative methods shown in Table II.

It has been observed and well documented in the past (and see e.g., Ref. [37]) that the CCM LSUBm results for this model (and many others) for both the gs energy $E$ and the sublattice magnetization $M$ show a distinct period-2 "staggering" effect with index $m$, according to whether $m$ is even or odd. As a consequence the LSUBm data for both $E$ and $M$ converge differently for the even- $m$ and the odd- $m$ sequences. This is very similar to what is also observed very frequently in perturbation theory in corresponding even and odd orders [45]. As a rule, therefore, the LSUBm data are generally extrapolated separately for even $m$ and for odd values of $m$, since the staggering makes extrapolations using both odd and even values together rather difficult. We show in Fig. 4 our LPSUB $m$ results for the gs energy per spin and the sublattice magnetization plotted against $1 / \mathrm{m}^{2}$ and $1 / m$, respectively, for the case $\Delta=1$. The higher odd and even $m$ values taken together clearly cluster well in both cases on straight lines, thereby justifying a posteriori our heuristic extrapolation fits of Eqs. (23) and (24). Just as in the LSUB $m$ case a small but definite "odd-even staggering" effect is observed in the LPSUB $m$ data for both the energy and the sublattice magnetization, although it is less pronounced than for the corresponding DSUB $m$ [33] and LSUB $m$ data [37] for this model.

Before discussing our LPSUBm results further for this model we note that the comparable LSUB $m$ solutions actually terminate at a critical value $\Delta_{c}=\Delta_{c}(m)$, which itself depends on the truncation index $m$ [32]. Such LSUB $m$ termination points are very common for many spin-lattice systems. They have been very well documented and their origin is clearly understood (and see e.g., Ref. [32]). Thus, in all such cases a termination point always arises due to the solution of the CCM equations becoming complex at this point, beyond which there 

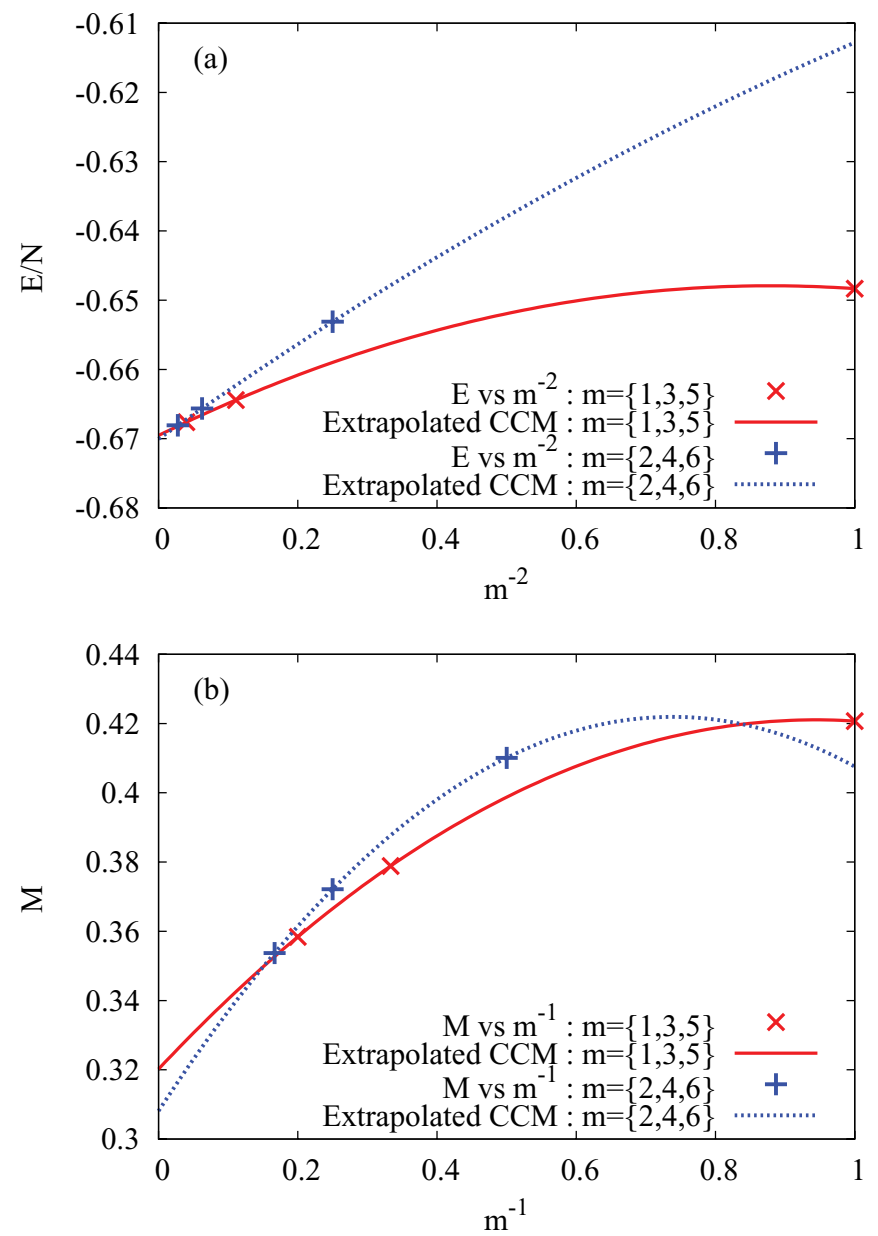

FIG. 4. (Color online) Illustration of the odd-even staggered nature with respect to the truncation parameter $m$ of the LPSUB $m$ scheme results for (a) the ground-state energy per spin, $E / N$, and (b) the sublattice magnetization, $M$, for the spin- $\frac{1}{2}$ antiferromagnetic $X X Z$ model on the 2D square lattice, for the isotropic limiting case $\Delta=1$. The LPSUB $m$ data are plotted against $1 / \mathrm{m}^{2}$ for $E / N$ and against $1 / m$ for $M$. The results clearly justify the heuristic extrapolation schemes of Eqs. (23) and (24).

exist two branches of entirely unphysical complex conjugate solutions [32]. In the region where the solution reflecting the true physical solution is real there actually also exists another (unstable) real solution. However, only the (shown) upper branch of these two solutions reflects the true (stable) physical GS, whereas the lower branch does not. The physical branch is usually easily identified in practice as the one which becomes exact in some known (e.g., perturbative) limit. This physical branch then meets the corresponding unphysical branch at the corresponding termination point, beyond which no real solutions exist. The LSUB $m$ termination points are themselves also reflections of the quantum phase transitions in the real system, and may hence be used to estimate the position of the phase boundary [32].

We note that when the LPSUB $m$ approximations are applied to the $X X Z$ model, only the odd LPSUB $m$ levels with $m \geqslant 3$ terminate in the same way as do the corresponding LSUBm approximations, as shown in Figs. 2 and 3. The LPSUBm solutions with even values $m=\{2,4,6\}$ do not terminate. We have no convincing explanation for this difference in behavior for two apparently similar schemes applied to the same model. Nevertheless, it is still possible to use our LPSUB $m$ data to extract an estimate for the physical phase transition point at which the $z$-aligned Néel phase terminates. As has been justified and utilized elsewhere [20], a point of inflexion at $\Delta=\Delta_{i}$ in the sublattice magnetization $M$ as a function of $\Delta$ also indicates the onset of an instability in the system. Such inflexion points $\Delta_{i}=\Delta_{i}(m)$ occur for the even values of the LPSUBm approximations, as indicated in Table II and Fig. 3. The LPSUB $m$ approximations are thus expected to be unphysical for $\Delta<\Delta_{i}(m)$, and we hence show the corresponding results for the gs energy per spin in Fig. 2 only for values $\Delta_{i}>\Delta_{i}(m)$. Heuristically, we find that the magnetization inflexion points $\Delta_{i}(m)$ scale linearly with $(1 / m)$ in the large $m$ limit, and the extrapolated results shown in Table II have been performed with $\Delta_{i}=c_{0}+c_{1}(1 / m)+c_{2}(1 / m)^{2}$, commensurate with the corresponding fits in $1 / \mathrm{m}^{2}$ and $1 / \mathrm{m}$ for the gs energy per spin and sublattice magnetization of Eqs. (23) and (24), respectively. The extrapolated values from both the LPSUB $m$ and DSUB $m$ schemes are in excellent agreement with the expected phase transition point at $\Delta_{c} \equiv 1$ between two quasiclassical Néel-ordered phases aligned along the spin $z$ axis (for $\Delta>1$ ) and in some arbitrary direction in the spin $x-y$ plane (for $|\Delta|<1$ ).

Although we do not do so here, the $x-y$ planar Néel phase could itself also easily be investigated by another CCM LPSUB $m$ series of calculations based on a model state $|\Phi\rangle$ with perfect Néel ordering in, say, the $x$ direction. Nevertheless, from our results so far we observe that the LPSUB $m$ scheme has, at least partially, fulfilled the expectations placed on it for the present model. Accordingly, we now apply it to the second test model of the spin- $\frac{1}{2} X Y$ model on the $2 \mathrm{D}$ square lattice.

\section{THE SPIN- $\frac{1}{2} X Y$ MODEL ON THE SQUARE LATTICE}

The Hamiltonian of the $X Y$ model [20] in global spin coordinates is written as

$$
H_{X Y}=\sum_{\langle i, j\rangle}\left[(1+\Delta) s_{i}^{x} s_{j}^{x}+(1-\Delta) s_{i}^{y} s_{j}^{y}\right], \quad-1 \leqslant \Delta \leqslant 1,
$$

where the sum on $\langle i, j\rangle$ again runs over all nearest-neighbor pairs of lattice sites and counts each pair only once. We again consider the case of spin- $\frac{1}{2}$ particles on each site of an infinite 2D square lattice.

For the classical model described by Eq. (28), it is clear that the GS is a Néel state in the $x$ direction for $0<\Delta \leqslant 1$ and a Néel state in the $y$ direction for $-1 \leqslant \Delta<0$. Hence, since we only consider the case $0 \leqslant \Delta \leqslant 1$, we choose as our CCM model state $|\Phi\rangle$ for the quantum $X Y$ model a Néel state aligned along the $x$ direction, written schematically as $|\Phi\rangle=$ $|\cdots \leftarrow \rightarrow \leftarrow \rightarrow \cdots\rangle$, in the global spin axes. Clearly the case $-1 \leqslant \Delta<0$ is readily obtained from the case $0<\Delta \leqslant 1$ by interchange of the $x$ and $y$ axes.

As in Sec. VI we now perform our usual rotation of the spin axes on each lattice site so that $|\Phi\rangle$ takes the form given by Eq. (17) in the rotated local spin coordinate frames. Thus, for the spins on the sublattice where they point in the negative $x$ 
direction in the global spin axes (i.e., the left-pointing spins) we perform a rotation of the spin axes by $+90^{\circ}$ about the spin $y$ axis. Similarly, for the spins on the other sublattice where they point in the positive $x$ direction in the global spin axes (i.e., the right-pointing spins) we perform a rotation of the spin axes by $-90^{\circ}$ about the spin $y$ axis. Under these rotations the spin operators are transformed for the left-pointing spins as

$$
s^{x} \rightarrow s^{z}, \quad s^{y} \rightarrow s^{y}, \quad s^{z} \rightarrow-s^{x},
$$

and for the right-pointing spins as

$$
s^{x} \rightarrow-s^{z}, \quad s^{y} \rightarrow s^{y}, \quad s^{z} \rightarrow s^{x} .
$$

The Hamiltonian of Eq. (28) may thus be rewritten in the local spin coordinate axes defined above as

$$
\begin{aligned}
H_{X Y}= & \sum_{\langle i, j\rangle}\left[-(1+\Delta) s_{i}^{z} s_{j}^{z}-\frac{1}{4}(1-\Delta)\left(s_{i}^{+} s_{j}^{+}+s_{i}^{-} s_{j}^{-}\right)\right. \\
& \left.+\frac{1}{4}(1-\Delta)\left(s_{i}^{+} s_{j}^{-}+s_{i}^{-} s^{+}\right)\right] .
\end{aligned}
$$

Exactly as in the previous application, we now have to evaluate the fundamental configurations that are retained in the CCM correlation operators $S$ and $\tilde{S}$ at each LPSUBm level of approximation. Although the point and space group symmetries of the square lattice (common to both the $X X Z$ and $X Y$ models considered here) and the two Hamiltonians of Eqs. (27) and (31) are identical, the numbers $N_{f}$ of fundamental configurations for a given LPSUB $m$ level are now larger (except for the case $m=1$ ) for the $X Y$ model than for the $X X Z$ model, since the uniform magnetization is no longer a good quantum number for the $X Y$ model, $\left[H_{X Y}, s_{T}^{z}\right] \neq 0$. Nevertheless, we note from the form of Eq. (31), in which the spin-raising and spin-lowering operators appear only in combinations that either raise or lower the number of spin flips by two (viz., the $s_{i}^{+} s_{j}^{+}$and $s_{i}^{-} s_{j}^{-}$combinations, respectively) or leave them unchanged (viz., the $s_{i}^{+} s_{j}^{-}$and $s_{i}^{-} s_{j}^{+}$combinations), it is only necessary for the $s_{T}^{z}=0 \mathrm{GS}$ to consider fundamental configurations that contain an even number of spins. Thus, the main difference for the $X Y$ model over the $X X Z$ model is that we must now also consider fundamental configurations in which we drop the restriction for the former case of having an equal number of spins on the two equivalent sublattices of the bipartite square lattice that was appropriate for the latter case. We show in Fig. 5 the fundamental configurations that are allowed for the spin- $\frac{1}{2} X Y$ model on the square lattice for the LPSUB $m$ approximation with $1 \leqslant m \leqslant 3$, and we invite the reader to compare with the corresponding

TABLE III. The ground-state energy per $\operatorname{spin}(E / N)$ and sublattice magnetization $(M)$ for the spin- $\frac{1}{2} X Y$ model on the $2 \mathrm{D}$ square lattice, obtained using the CCM LPSUB $m$ approximation scheme with $1 \leqslant m \leqslant 6$ at $\Delta=0 . N_{f}$ is the number of fundamental configurations at a given level of LPSUB $m, \mathrm{LSUB} m$, or DSUB $m$ approximation. $\Delta_{c} \equiv \mathrm{LPSUB} m$ termination point. The LPSUB $m$ results for odd values

\begin{tabular}{|c|c|c|c|c|c|}
\hline Method & $N_{f}$ & $\begin{array}{c}E / N \\
(\Delta=0)\end{array}$ & $\begin{array}{c}M \\
(\Delta=0)\end{array}$ & $\Delta_{c}$ & $\begin{array}{c}\text { Max. No. } \\
\text { of Spins }\end{array}$ \\
\hline LPSUB1 & 1 & -0.54031 & 0.475 & a & 2 \\
\hline LPSUB2 & 4 & -0.54548 & 0.464 & -0.401 & 4 \\
\hline LPSUB3 & 13 & -0.54747 & 0.457 & -0.178 & 6 \\
\hline LPSUB4 & 72 & -0.54812 & 0.453 & -0.107 & 8 \\
\hline LPSUB5 & 557 & -0.54842 & 0.450 & -0.072 & 12 \\
\hline LPSUB6 & 7410 & -0.54857 & 0.448 & b & 16 \\
\hline LSUB6 & 131 & -0.54833 & 0.451 & -0.073 & 6 \\
\hline LSUB8 & 2793 & -0.54862 & 0.447 & -0.04 & 8 \\
\hline SE & & -0.5488 & 0.436 & 0.0 & \\
\hline \multirow[t]{2}{*}{ QMC } & & $-0.54882(2)$ & $0.437(2)$ & & \\
\hline & & Extrapolation & & & Based on \\
\hline LPSUB $\infty$ & & -0.54894 & 0.437 & -0.017 & $2 \leqslant m \leqslant 5$ \\
\hline LPSUB $\infty$ & & -0.54897 & 0.435 & -0.006 & $3 \leqslant m \leqslant 5$ \\
\hline LPSUB $\infty$ & & -0.54893 & 0.436 & b & $2 \leqslant m \leqslant 6$ \\
\hline LPSUB $\infty$ & & -0.54894 & 0.435 & $\mathrm{~b}$ & $3 \leqslant m \leqslant 6$ \\
\hline LPSUB $\infty$ & & -0.54888 & 0.436 & $\mathrm{~b}$ & $4 \leqslant m \leqslant 6$ \\
\hline LPSUB $\infty$ & & -0.54899 & 0.437 & $\mathrm{a}$ & $m=\{1,3,5\}$ \\
\hline LPSUB $\infty$ & & -0.54893 & 0.436 & $\mathrm{~b}$ & $m=\{2,4,6\}$ \\
\hline $\mathrm{LSUB} \infty$ & & -0.54892 & 0.435 & 0.00 & $m=\{4,6,8\}$ \\
\hline $\mathrm{DSUB} \infty$ & & -0.54950 & 0.436 & & $m=\{3,5,7,9\}$ \\
\hline DSUB $\infty$ & & -0.54923 & 0.437 & 0.011 & $m=\{5,7,9\}$ \\
\hline
\end{tabular}
of $m$, even values of $m$, and the whole series of $m$ are extrapolated separately. These results are compared to calculations using series expansions (SE) [46], the quantum Monte Carlo (QMC) method [47], LSUB $\infty$ extrapolations of the CCM LSUB $m$ approximations [20], and the DSUB $\infty$ extrapolations of the CCM DSUB $m$ approximations [33].

\footnotetext{
${ }^{a}$ The LPSUB1 approximation does not terminate.

${ }^{\mathrm{b}}$ The spin-flip configurations for the LPSUB6 approximation are sufficiently complicated and large in number that calculations have only been calculated at present for $\Delta=0$ for the LPSUB6 case.
} 


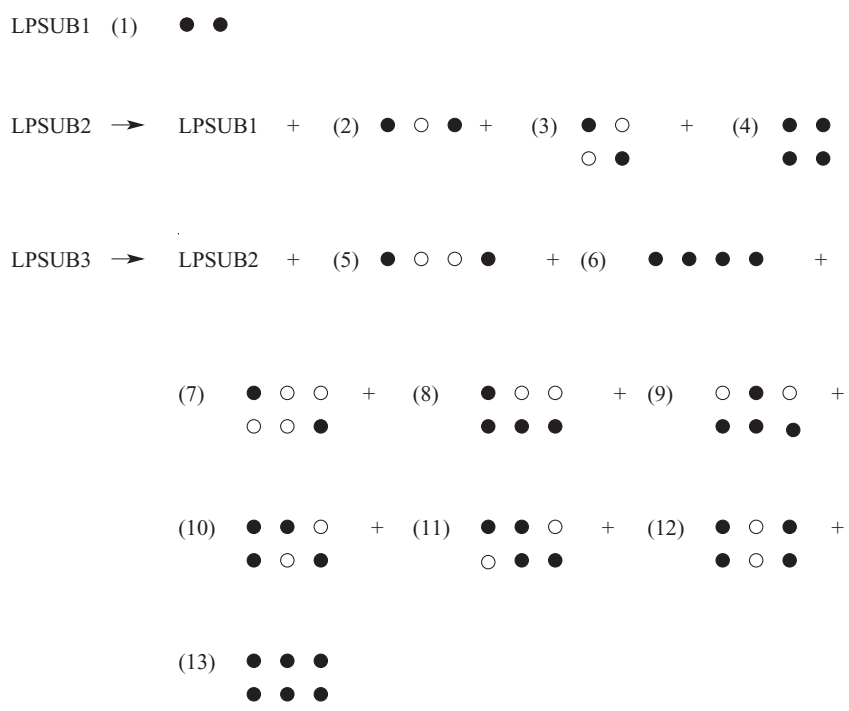

FIG. 5. The fundamental configurations for the LPSUB $m$ scheme with $m=\{1,2,3\}$ for the spin- $\frac{1}{2} X Y$ model on a square lattice in two dimensions. The filled circles mark the relative positions of the sites of the square lattice on which the spins are flipped with respect to the model state. The unfilled circles represent unflipped sites.

fundamental configurations for the spin- $\frac{1}{2} X X Z$ model on the same square lattice shown in Fig. 1. The corresponding numbers $N_{f}$ of fundamental configurations for the $X Y$ model are also shown in Table III for the LPSUB $m$ approximations with $m \leqslant 6$.

We present results for the spin- $\frac{1}{2} X Y$ model on the square lattice in the CCM LPSUB $m$ approximations for all values $m \leqslant 6$, all of which can be computed with reasonably modest computing power. Comparable computing power enables the corresponding $\mathrm{LSUB} m$ scheme to be implemented for all $m \leqslant 8$. Numerical results for the gs energy per spin and sublattice magnetization are shown in Table III at the isotropic

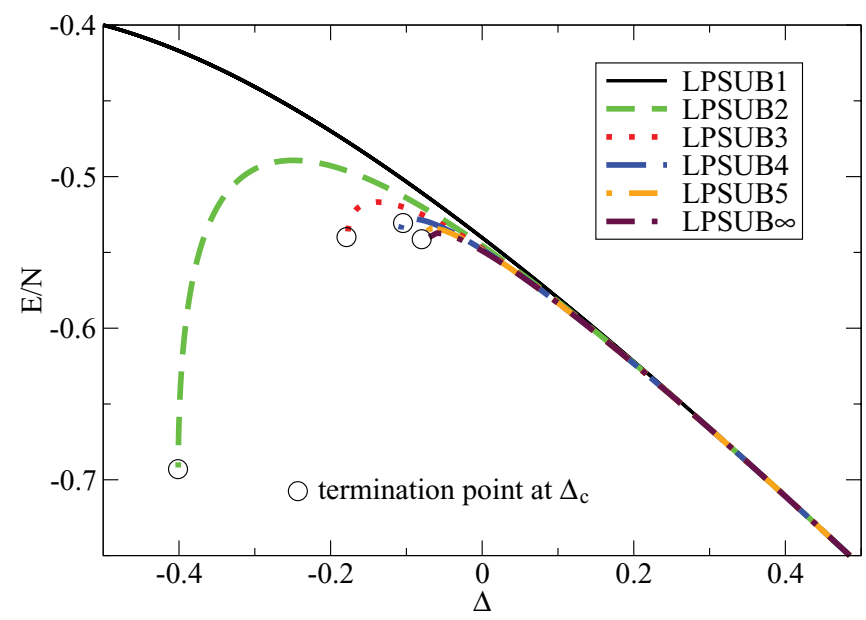

FIG. 6. (Color online) CCM results for the ground-state energy per spin, $E / N$, as a function of the anisotropy parameter $\Delta$, of the spin- $\frac{1}{2} X Y$ model on the $2 \mathrm{D}$ square lattice obtained using the LPSUB $m$ approximation based on the Néel state aligned along any axis in the $x-y$ plane. The LPSUB $m$ results with $m=\{1,3,5\}$ are extrapolated using Eq. (23) to give the curve labeled LPSUB $\infty$.

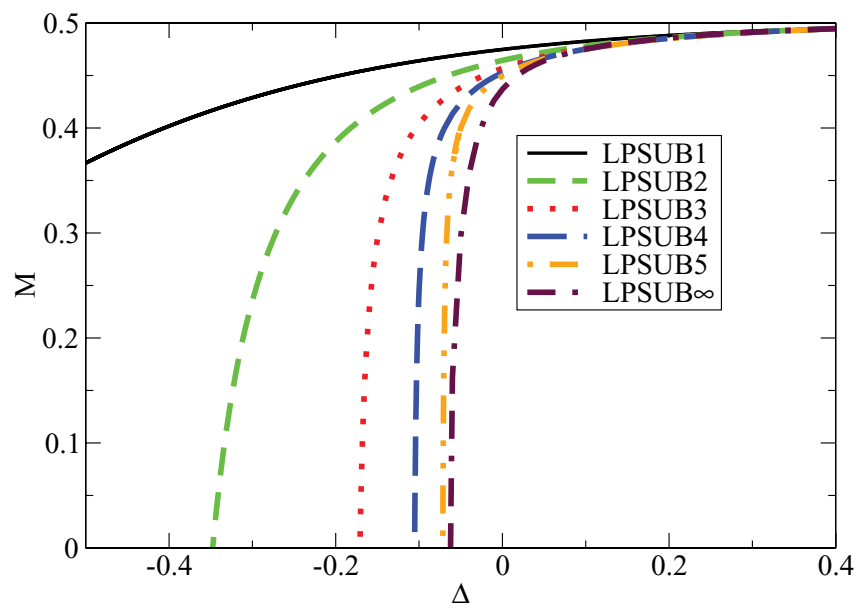

FIG. 7. (Color online) CCM results for the ground-state sublattice magnetization, $M$, as a function of the anisotropy parameter $\Delta$, of the spin- $\frac{1}{2} X Y$ model on the $2 \mathrm{D}$ square lattice obtained using various LPSUB $m$ approximations based on the Néel state aligned along any axis in the $x-y$ plane. The LPSUB $m$ results with $m=\{1,3,5\}$ are extrapolated using Eq. (24) to give the curve labeled LPSUB $\infty$.

point at $\Delta=0$ at various levels of approximation, and corresponding results for the same gs quantities are shown graphically in Figs. 6 and 7 as functions of the anisotropy parameter $\Delta$.

We also show in Table III for the isotropic $X Y$ Hamiltonian $(\Delta=0)$ the results for the gs energy and sublattice magnetization using the (quadratic) extrapolation schemes of Eqs. (23) and (24), respectively, of the LPSUB $m$ data, employing various subsets of our results, just as for the $X X Z$ model considered previously. We also compare in Table III the present results with the corresponding CCM LSUB $m$ [20] and DSUB $m$ [33] results for the same model. All of the CCM results are clearly in excellent agreement both with one another and with the results of the best of the alternative methods available for this model, including the linked-cluster series expansion (SE) technique [46] and a quantum Monte Carlo (QMC) method [47].

We show in Fig. 8 our LPSUB $m$ results for the present $X Y$ model for the gs energy per spin and the sublattice magnetization, plotted respectively against $1 / \mathrm{m}^{2}$ and $1 / \mathrm{m}$, for the case $\Delta=0$. As previously for the $X X Z$ model, the higher $m$ values cluster well on straight lines in both cases, thereby justifying once more our heuristic choice of extrapolation fits indicated in Eqs. (23) and (24). Figures 8(a) and 8(b) once more show an "odd-even" staggering effect in the termination index $m$ for the LPSUB $m$ data and we have again shown separate extrapolations of our LPSUB $m$ results in Table III for the even- $m$ data and the odd- $m$ data, as well as results using all (higher) values of $m$. It is interesting to note, however, that the staggering effect for this $X Y$ model is far less pronounced than for the similar $X X Z$ model in Sec. VI. We have no compelling argument to explain this difference.

It is interesting to note that for the present $X Y$ model the CCM LPSUB $m$ solutions (with our choice of model state as a Néel state in the $x$ direction) now do physically terminate for all values of the truncation index $m \geqslant 1$ at a critical value $\Delta_{c}=\Delta_{c}(m)$, exactly as commonly occurs (as for the present 

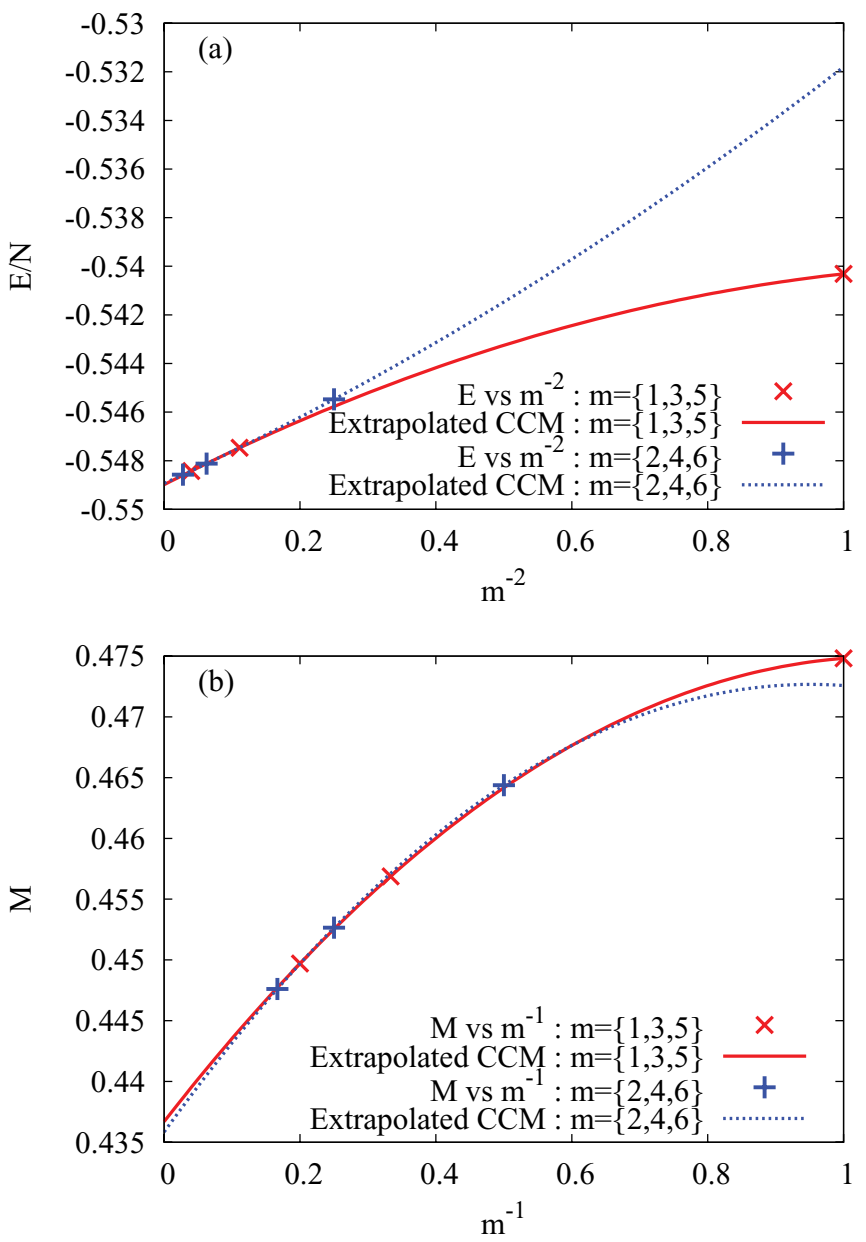

FIG. 8. (Color online) Illustration of the odd-even staggered nature with respect to the truncation parameter $m$ of the LPSUB $m$ scheme results for (a) the ground-state energy per spin, $E / N$, and (b) the sublattice magnetization, $M$, for the spin- $\frac{1}{2} X Y$ model on the 2D square lattice, for the isotropic limiting case $\Delta=0$. The LPSUB $m$ data are plotted against $1 / \mathrm{m}^{2}$ for $E / N$ and against $1 / m$ for $M$. The results clearly justify the heuristic extrapolation schemes of Eqs. (23) and (24).

model) for the LSUBm calculations, as we explained above in Sec. VI. Why such LPSUBm terminations occur for all values $m>1$ for the $X Y$ model but not for odd values of $m$ for the previous $X X Z$ model is not obvious to us. The corresponding termination points, $\Delta_{c}=\Delta_{c}(m)$, at various LPSUB $m, \mathrm{LSUB} m$, and DSUB $m$ levels of approximation are shown in Table III. It has been shown previously [31] that $\Delta_{c}(m)$ scales well with $(1 / m)^{2}$ for the LSUBm data, and the $\mathrm{LSUB}_{\infty} \infty$ result [20] shown in Table III was obtained by the scaling law $\Delta_{c}(m)=d_{0}+d_{1}(1 / m)^{2}+d_{2}(1 / m)^{4}$. We find heuristically that the best large- $m$ asymptotic behavior of the LPSUB $m$ data for $\Delta_{c}(m)$ is also against $(1 / m)^{2}$ as the scaling parameter. Accordingly, the LPSUB $\infty$ values for $\Delta_{c}$ in Table III are obtained with the same (quadratic) fit, $\Delta_{c}(m)=d_{0}+d_{1}(1 / m)^{2}+d_{2}(1 / m)^{4}$. We see that both the $\mathrm{LSUB} \infty$ and LPSUB $\infty$ results for $\Delta_{c} \equiv \Delta_{c}(\infty)$ agree very well with the value $\Delta_{c}=0$ that is known to be the correct value for the phase transition in the one-dimensional spin- $\frac{1}{2}$
$X Y$ chain from the known exact solution [48], and which is believed on symmetry grounds also to be the phase transition point for higher dimensions, including the present 2D square lattice.

\section{CONCLUSIONS}

From the two nontrivial benchmark spin-lattice problems that we have investigated here, it is clear that the new LPSUB $m$ approximation scheme works well for calculating their gs properties and phase boundaries. We have utilized here only the simplest extrapolation schemes in the pertinent scaling variables, and have shown that these may be chosen, for example, as $1 / m^{2}$ for the gs energy and $1 / m$ for the order parameter. For further use of the scheme for more complex lattice models (e.g., those exhibiting geometric or dynamic frustration) it will be necessary to revisit the validity of these expansions, but a great deal of previous experience in such cases for the LSUBm scheme should provide good guidance.

On the basis of the test results presented here, the LPSUB $m$ scheme clearly does not fulfill the first of our two main criteria for introducing it, since the number of fundamental configurations, $N_{f}$, actually increases even more rapidly with truncation index $m$ than for the corresponding LSUB $m$ series of approximations. Nevertheless, our second criterion of capturing the physically most important configurations at relatively low levels of approximation does seem to be fulfilled, according to our experience with the convergence of the LPSUBm sequences for observable quantities. At the very least we now have three schemes (LSUBm, DSUB $m$, and $\mathrm{LPSUB} m$ ) available to us for future investigations of more complicated spin-lattice models, each of which has its own merits, and which thus allows us more freedom in future applications of the CCM to quantum magnetism.

It is particularly worth noting too that our preliminary calculations have shown that the different schemes show markedly varying patterns of odd-even staggering, both for a given scheme applied to different models and for different schemes applied to the same model. It is difficult to predict in advance how strong or weak the effect will be for a given scheme applied to a specific model. Nevertheless, when the effect is weak one can confidently extrapolate the results using both odd and even values of the truncation parameter $m$ simultaneously, thereby effectively doubling the number of data points for the fit. In such cases our first criterion for an improved scheme has effectively been realized over one where the staggering effect is much more pronounced, even though the number of fundamental configurations, $N_{f}$, may indeed increase more rapidly with truncation index $m$ for the former ("improved") scheme than for the latter.

\section{ACKNOWLEDGMENTS}

We are grateful to Dr. J. Schulenburg of Universität Magdeburg for his assistance in the incorporation into the CCM computer code of the LPSUB $m$ approximation scheme. 
[1] F. Coester, Nucl. Phys. 7, 421 (1958).

[2] J. Č́ížek, J. Chem. Phys. 45, 4256 (1966).

[3] J. Paldus, J. Č́́žek, and I. Shavitt, Phys. Rev. A 5, 50 (1972).

[4] H. Kümmel, K. H. Lührmann, and J. G. Zabolitzky, Phys. Rep. C 36, 1 (1978).

[5] J. S. Arponen, Ann. Phys. (NY) 151, 311 (1983).

[6] J. S. Arponen, R. F. Bishop, and E. Pajanne Phys. Rev. A 36, 2539 (1987).

[7] R. J. Bartlett, J. Phys. Chem. 93, 1697 (1989).

[8] R. F. Bishop, Theor. Chim. Acta 80, 95 (1991).

[9] R. F. Bishop, in Microscopic Quantum Many-Body Theories and Their Applications, Lecture Notes in Physics 510, edited by J. Navarro and A. Polls (Springer-Verlag, Berlin, 2004), p. 1.

[10] R. F. Bishop and K. H. Lührmann, Phys. Rev. B 17, 3757 (1978).

[11] R. F. Bishop and K. H. Lührmann, Phys. Rev. B 26, 5523 (1982).

[12] K. Emrich and J. G. Zabolitzky, Phys. Rev. B 30, 2049 (1984).

[13] B. D. Day, Phys. Rev. Lett. 47, 226 (1981).

[14] B. D. Day and J. G. Zabolitzky, Nucl. Phys. A 336, 221 (1981).

[15] R. J. Bartlett, Annu. Rev. Phys. Chem. 32, 359 (1981).

[16] D. M. Ceperley and B. J. Alder, Phys. Rev. Lett. 45, 566 (1980).

[17] M. Roger and J. H. Hetherington, Phys. Rev. B 41, 200 (1990).

[18] R. F. Bishop, J. B. Parkinson, and Yang Xian, Phys. Rev. B 44, 9425 (1991).

[19] R. Bursill, G. A. Gehring, D. J. J. Farnell, J. B. Parkinson, T. Xiang, and C. Zeng, J. Phys. Condens. Matter 7, 8605 (1995).

[20] D. J. J. Farnell, S. E. Krüger, and J. B. Parkinson, J. Phys. Condens. Matter 9, 7601 (1997).

[21] R. F. Bishop, D. J. J. Farnell, and J. B. Parkinson, Phys. Rev. B 58, 6394 (1998).

[22] R. F. Bishop, D. J. J. Farnell, S. E. Krüger, J. B. Parkinson, J. Richter, and C. Zeng, J. Phys. Condens. Matter 12, 6887 (2000).

[23] D. J. J. Farnell, K. A. Gernoth, and R. F. Bishop, Phys. Rev. B 64, 172409 (2001).

[24] D. J. J. Farnell, R. F. Bishop, and K. A. Gernoth, J. Stat. Phys. 108, 401 (2002).

[25] R. F. Bishop, P. H. Y. Li, R. Darradi, and J. Richter, J. Phys. Condens. Matter 20, 255251 (2008).

[26] R. F. Bishop, P. H. Y. Li, R. Darradi, and J. Richter, Europhys. Lett. 83, 47004 (2008).

[27] R. F. Bishop, P. H. Y. Li, R. Darradi, J. Schulenburg, and J. Richter, Phys. Rev. B 78, 054412 (2008).
[28] R. F. Bishop, P. H. Y. Li, R. Darradi, J. Richter, and C. E. Campbell, J. Phys. Condens. Matter 20, 415213 (2008).

[29] R. F. Bishop, P. H. Y. Li, D. J. J. Farnell, and C. E. Campbell, Phys. Rev. B 79, 174405 (2009).

[30] R. F. Bishop, J. B. Parkinson, and Yang Xian, Phys. Rev. B 43, 13782 (1991).

[31] R. F. Bishop, R. G. Hale, and Yang Xian, Phys. Rev. Lett. 73, 3157 (1994).

[32] D. J. J. Farnell and R. F. Bishop, in Quantum Magnetism, Lecture Notes in Physics 645, edited by U. Schollwöck, J. Richter, D. J. J. Farnell, and R. F. Bishop (Springer-Verlag, Berlin, 2004), p. 307.

[33] R. F. Bishop, P. H. Y. Li, and J. Schulenburg, Condens. Matter Phys. 12, 479 (2009).

[34] C. Zeng, D. J. J. Farnell, and R. F. Bishop, J. Stat. Phys. 90, 327 (1998).

[35] S. E. Krüger, J. Richter, J. Schulenburg, D. J. J. Farnell, and R. F. Bishop, Phys. Rev. B 61, 14607 (2000).

[36] D. Schmalfuß, R. Darradi, J. Richter, J. Schulenburg, and D. Ihle, Phys. Rev. Lett. 97, 157201 (2006).

[37] D. J. J. Farnell and R. F. Bishop, Int. J. Mod. Phys. B 22, 3369 (2008).

[38] J. Richter, R. Darradi, R. Zinke, and R. F. Bishop, Int. J. Mod. Phys. B 21, 2273 (2007).

[39] C. J. Hamer, Zheng Weihong, and P. Arndt, Phys. Rev. B 46, 6276 (1992).

[40] Zheng Weihong, J. Oitmaa, and C. J. Hamer, Phys. Rev. B 43, 8321 (1991).

[41] J. Richter, J. Schulenburg, and A. Honecker, in Quantum Magnetism, Lecture Notes in Physics 645, edited by U. Schollwöck, J. Richter, D. J. J. Farnell, and R. F. Bishop (Springer-Verlag, Berlin, 2004), p. 85.

[42] A. W. Sandvik, Phys. Rev. B 56, 11678 (1997).

[43] U. Löw, Phys. Rev. B 76, 220409(R) (2007).

[44] T. Kennedy, E. H. Lieb, and S. Shastry, J. Stat. Phys. 53, 1019 (1988).

[45] P. M. Morse and H. Feshbach, Methods of Theoretical Physics, Part II (McGraw-Hill, New York, 1953).

[46] C. J. Hamer, J. Oitmaa, and Zheng Weihong, Phys. Rev. B 43, 10789 (1991).

[47] A. W. Sandvik and C. J. Hamer, Phys. Rev. B 60, 6588 (1999).

[48] E. Lieb, T. Schultz, and D. Mattis, Ann. Phys. (NY) 16, 407 (1961). 